\title{
Naming babies "Baby-of" at birth: A project to encourage earlier naming of infants in Abu Dhabi
}

Rodney Jay Santos, Luena Palacios, Rommel Amuan

Ambulatory Healthcare Services-SEHA - Abu Dhabi, U.A.E.

\begin{abstract}
In 2013, Sweihan Healthcare Center, one of several government clinics in Abu Dhabi, catered to 19,349 patients or an average of 52 patients in a day. During the weekdays, close to 80 patients are seen per day, a relatively huge number for a small town clinic. In 2011 , the clinic only saw 16,816 patients, which amounts to a $15 \%$ increase in two years.
\end{abstract}

Circumstances called for a search for gaps in the system in order to ease the workflow of a steadily increasing patient influx. The focus was mainly on patient identification due to a considerable number of patients having the same name. Data extraction was simplified by the advent of electronic medical records and, as the names of the patients were filtered, one name stood out: "Baby of".

The goal of this project was to track the patients using the name "Baby of", and change their names in their respective medical records and thus be able to comply with the International Patient Safety Goals (IPSG) of both Joint Commission International [1] and the Health Authority of Abu Dhabi [2].

\section{Problem}

In this country certain names are very popular. As a result there is a risk of calling out a patient's name for treatment and giving incorrect treatment to a patient who has the same name. In hospitals until a new baby is given a name by their parents they are given the name " "Baby of" followed by the parents' surname, and as many surnames are the same, this results in higher risks of giving incorrect treatment to a baby, especially one born on the same day as another with the same name. Many babies then leave hospital without a first name increasing the risk of similar problems in future.

\section{Background}

"Baby of" is the default name given to an infant until the parents have decided on what name they will give their child.

Before this project, the authors are not aware of any campaign in place to track down the patients still named "Baby of".

Many parents fail to come up with a name or names to be given to their child/children during, prior, and even after pregnancy. After the baby is born many parents still do not know what name to call their baby before discharge from hospital, so paperwork and health records continue to say "Baby of" (followed by surname).

\section{Baseline measurement}

The first measure taken was to ascertain the prevalence, initially in the remote town of Sweihan, of patients registered as "Baby of". Through data extraction in Malaffi / Cerner, (the healthcare information/documentation system that is utilized across all
Ambulatory Healthcare Service (AHS) entities in Abu Dhabi), the reference data was gathered. In 2013, the year the data was gathered; it was found that the clinic has 174 patients registered as "Baby of" (Baby of, B/O, or BO). Alarmingly, out of these 174 patients, 137 or $79 \%$ of them were already between 1 to 7 years of age.

See supplementary file: ds4346.pptx - "Baseline data of "Baby of" Patients"

\section{Design}

With the cooperation with the Health Information Management team (HIM), Sweihan Clinic's quality improvement team thought of a way to raise the issue and create awareness by presenting this case as a Quality Improvement Project presentation during AHS's Quality week. As a result the issue was brought to the attention of senior management of the organisation.

\section{Strategy}

PDSA Cycle 1:

The first step was to contact parents of children named "Baby of". The task of communicating with the parents of the "Baby-of" patients was primarily assigned to the Patient access representatives (front-desk staff), because they could speak the local language and could readily persuade the parents to submit a number of documents to start the name change.

The following documents were asked for: (1) Child's Birth Certificate, (2) National ID (Emirates ID), (3) Passport and Visa 
copy / Family Book (for local nationals), and (4) the Health Card to enable the HIM to start the process. Expectations for a high turnout were anticipated, however, contrary to optimistic assumptions, the project only managed to change 52 out of 174 patients' names, or $30 \%$, on the initial run.

PDSA Cycle 2:

It appeared that parents were not convinced of the importance of changing their child's name officially. It was then decided that the healthcare professionals (HCP), mainly the physicians and the nurses, were to assist the front desk staff by pointing out the implications of not changing the names of their children as part of the discussion with parents, focussing on the risks of inadequate treatment as a result of people having the same name, and the identification issues in future.

PDSA Cycle 3:

It was noted that documents were not being returned causing delays in the project, so the AHS Quality team aided the project by introducing the E-Service "Demographic Change Request", which can be utilised electronically by any AHS staff through the company intranet. The service allows scanning and instant uploading of the aforementioned documents rather than sending a copy through courier, hence, expediting the process.

Data was re-evaluated in comparison to the first PDSA after 6 months.

\section{Results}

Post evaluation data revealed that out of the 174 patients, $82 \%$ (143) of patients have had their names changed in the system, a remarkable improvement to the previous 30\% turnout from the initial run. Out of the 174 patients, $10 \%$ (17) remaining are still pending in Health Information Management, 3\% (5) still failed to submit their child's documents and 5\% (9) are newborns and are newly registered to the clinic.

See supplementary file: ds4347.jpg - "Post Evaluation Data"

\section{Lessons and limitations}

As rigorously as the staff are working on getting a hold of the "Baby Of" patients, it was found that to be able to persuade the parents of these patients, the benefits (and the possible repercussions) must be carefully explained to encourage them to participate. And even with all that, there are still some parents that are still unwilling to participate in this culture changing endeavour.

\section{Conclusion}

Ultimately, the task of eliminating "Baby of" names would be relatively simple if teamwork existed between all concerned parties including parents and the healthcare professionals. As it is advised for mothers to have regular check-ups during pregnancy and after birth, the task of reminding the mother and her husband to start contemplating a name for their child or children should fall on the hands of the attending physician. The available technology is capable of determining the gender of a baby months before delivery.

As for the existing "Baby of" patients, parents should be sought out, and encouraged to have their child's name changed in the system. As previously mentioned, only a handful of documents are needed to be brought to their nearest local clinic, and healthcare workers there are always prepared to fulfill this duty.

\section{References}

1 Alexander D, Costa Jr. H, Devolder B, Ellaham S, Ghoneim H, Hoffman $\mathrm{P}$, et al, Joint Commission International Accreditation Standards for Ambulatory Care, 3rd Edition; 2015: 20[accessed 18 November 2014]. Available from:

http://www.jointcommissioninternational.org/jci-accreditation-standa rds-for-ambulatory-care-3rd-edition-english-version-pdf-book-/

2 Matear D, HAAD Standards for Licensure and Quality Improvement: 10 [Accessed 18 November 2014]. Available from: http://www.haad.ae/HAAD/Portals/0/Referal files/referals/Accident \%20Emergency\%20Care\%20Related\%20Standards.pdf

\section{Declaration of interests}

Nothing to declare

\section{Acknowledgements}

Dr. Kamal Babiker (Clinic Manager), Mr. Mohammed Nasser Al Mazrouei (Clinic Administrator), Mr. Rommel C. Amuan, RN (Clinic Head Nurse), Ms. Luena Palacios (Senior Quality Officer), Dr. Fayeza Saif Nasir (Director of Quality - AHS), Mr. Mohammed Hawas AI Sadid (CEO - AHS), Dr. Omar Naseeb Al Jabri (Chief Medical Officer - AHS), Ms. Louise Bain (Chief Operating Officer AHS) 